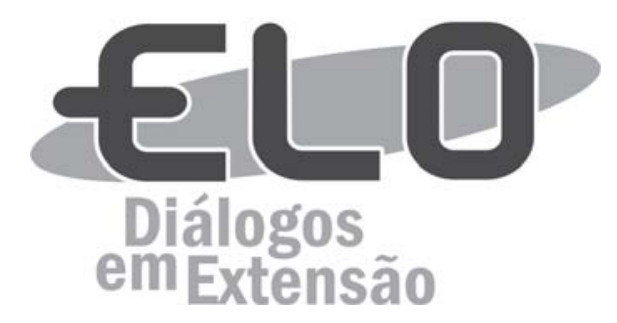

\title{
"O que sabemos sobre cuidados paliativos": (re)construindo conceitos por meio de uma experiência dialógica
}

\author{
João Vitor Andrade ${ }^{1}$, Ana Luiza Rodrigues Lins², Letícia Milagres \\ Paiva ${ }^{3}$, Thalyta Cássia de Freitas Martins ${ }^{4}$ e Erica Toledo de Mendonça ${ }^{5}$
}

\begin{abstract}
Resumo: Este relato apresenta como objeto uma dinâmica que identificou conhecimentos prévios dos participantes sobre cuidados paliativos. Tem como objetivo relatar a experiência e os resultados da dinâmica "O que sabemos sobre cuidados paliativos?", realizada pela Liga de Oncologia no minicurso de cuidados paliativos, e é um estudo descritivo do tipo relato de experiência. No primeiro momento da atividade, os participantes responderam por escrito à questão "O que sabemos sobre cuidados paliativos?". Após, os mesmos colaram as tarjetas na lousa para posterior debate. As respostas foram avaliadas pela análise de conteúdo. Emergiram três categorias; uma que conceituou o tema, relacionando-o às tecnologias leves, e as demais, que expressavam os entraves, dilemas e potencialidades dos cuidados paliativos. Destaca-se a importância de implementar estratégias como a descrita, a fim de discutir e ressignificar os cuidados paliativos entre estudantes e profissionais de saúde.
\end{abstract}

Palavras-chave: Capacitação de Recursos Humanos em Saúde. Cuidados Paliativos. Neoplasias. Relações Comunidade-Instituiçôes.

Área Temática: Saúde.

\section{"What we know about palliative care": (re) constructing concepts through a dialogic experience}

Abstract: This report presents as an object a dynamics that identified participants prior knowledge about palliative care. Its purpose is to report on the experience and results of the of the dynamics "What do we know about palliative care?" carried out by the League of Oncology in the mini course of palliative care and is a descriptive study the type experience report. At the first moment of the activity, the participants answered in writing to the question, 'What do we know about palliative care?' After that, they pasted the cards on the board for posterior discussion. Responses were analyzed by content analysis. Three categories emerged, one that conceptualized the theme, relating it to light technologies, and the others expressing the obstacles, dilemmas and potentialities of palliative care. Stands out it is important to implement strategies such as the one described in order to discuss and re-signify the palliative care among students and health professionals.

Keywords: Community-Institutional Relations. Health Human Resource Training. Neoplasms. Palliative Care.

\footnotetext{
${ }^{1}$ Acadêmico do curso de graduação em Enfermagem da Universidade Federal de Viçosa, Minas Gerais. Avenida PH Rolphs, Departamento de Medicina e Enfermagem, s/n, Campus Universitário, Universidade Federal de Viçosa, MG. Telefone: 35-99856-8746. E-mail: jvma100@gmail.com

${ }^{2}$ Acadêmica do curso de graduação em Enfermagem da Universidade Federal de Viçosa, Minas Gerais.

${ }^{3}$ Acadêmica do curso de graduação em Psicologia da UNIVIÇOSA, Viçosa, Minas Gerais.

${ }^{4}$ Docente do curso de graduação em Enfermagem do Departamento de Medicina e Enfermagem da Universidade Federal de Viçosa, Minas Gerais.

${ }^{5}$ Docente do curso de graduação em Enfermagem do Departamento de Medicina e Enfermagem da Universidade Federal de Viçosa, Minas Gerais. Coordenadora da Liga Acadêmica de Oncologia- Karkinos.
} 


\section{"Lo que sabemos sobre cuidados paliativos": (re) construcción de conceptos a través de una experiencia dialógica}

Resumen: Este informe presenta como un objeto una dinámica que identificó el conocimiento previo de los participantes sobre los cuidados paliativos. Tiene como objetivo: reportar la experiencia y los resultados de la dinámica "Qué sabemos sobre los cuidados paliativos?" realizado por la Liga de Oncología en el minicurso de cuidados paliativos y es uno estudio descriptivo del tipo informe de experiencia. En el primer momento de la actividad, los participantes respondieron por escrito a la pregunta 'Qué sabemos sobre los cuidados paliativos?' Después de eso, colocaron las cartas en la pizarra para un debate posterior. Las respuestas fueron analizadas por el análisis de contenido. Surgieron tres categorías, una que conceptuaba el tema, relacionándolo con las tecnologías ligeras, y los demás expresan los obstáculos, dilemas y potencialidades de los cuidados paliativos. Se resalta la importancia de la implementación de estrategias como la descrita para discutir y resignificar cuidados paliativos entre estudiantes y profesionales de salud.

Palabras clave: Capacitación de Recursos Humanos en Salud. Cuidados Paliativos. Neoplasias. Relaciones Comunidad-Institución.

\section{Introdução}

O Brasil tem passado pelo processo de transição epidemiológica, caracterizado pelo decréscimo das taxas de mortalidade por doenças infecto parasitárias e aumento progressivo dos óbitos decorrentes das doenças e agravos não transmissíveis (DELALIBERA et al., 2016). Nesse contexto, o câncer se impõe como a segunda causa de morte, perdendo apenas para as doenças cardiovasculares. Segundo a Organização Mundial de Saúde (OMS, 2017), as neoplasias representaram 21\% dos 36 milhões de óbitos ocorridos em 2012.

Dados do Instituto Nacional do Câncer revelam que até 2030 espera-se a ocorrência de aproximadamente 30 milhões de novos casos de câncer no mundo. Seguindo essa tendência mundial, no Brasil ocorrerão cerca de 640 mil novos casos por ano no biênio 2018/2019 (INCA, 2018). Logo, as neoplasias representam um importante problema mundial de saúde pública, sendo de suma importância o investimento em políticas públicas de prevenção e apoio à pessoa com câncer.

Destaca-se que uma das formas de assistência à pessoa com neoplasias são os cuidados paliativos, sendo estes definidos pela International Association for Hospice e Palliative Care (IAHPC, 2019) como:

\footnotetext{
Assistência promovida por uma equipe multidisciplinar, que objetiva a melhoria da qualidade de vida do paciente e seus familiares, diante de uma doença que ameace a vida, por meio da prevenção e alívio do sofrimento, da identificação precoce, avaliação impecável e tratamento de dor e demais sintomas físicos, sociais, psicológicos e espirituais (IAHPC, 2019, p.1).
}

Nessa perspectiva, a KARKINOS - Liga Acadêmica de Oncologia, composta atualmente por discentes dos cursos de Psicologia, Enfermagem e Medicina, realizou a dinâmica intitulada: "O que sabemos sobre cuidados paliativos?", durante um minicurso de cuidados paliativos realizado na referida universidade em setembro de 2018.

Assim, este estudo tem por finalidade relatar a experiência e os resultados da dinâmica "O que sabemos sobre cuidados paliativos?" realizada pela Liga de Oncologia durante um minicurso de cuidados paliativos.

\section{Métodos}

Trata-se de um estudo descritivo do tipo relato de experiência, realizado por membros da KARKINOS. A dinâmica aqui relatada, que objetivou entender o que os participantes do minicurso sabiam sobre o tema, foi o primeiro momento do minicurso de Cuidados Paliativos. O mesmo foi realizado no dia 28 de setembro de 2018 na Universidade Federal de Viçosa e contou com a presença de 50 pessoas, dentre estudantes e profissionais de Nutrição, Enfermagem, Medicina e Psicologia. 
A dinâmica compreendeu dois momentos. No primeiro momento os participantes foram esclarecidos acerca da proposta, na qual teriam que responder à seguinte comanda inscrita na tarjeta, rememorando seus conhecimentos prévios: “O que sabemos sobre cuidados paliativos?", com apenas uma frase ou palavra, num tempo de 10 minutos.

Posteriormente, os mesmos colaram suas tarjetas na lousa, e, após esse momento, os facilitadores da dinâmica realizaram a leitura e análise de cada conceito apresentado, dialogando com os participantes, a fim de estimular a reflexão e o debate sobre as respostas elencadas pelos participantes.

Após encerramento da dinâmica, os autores recolheram as tarjetas e realizaram a análise dos dados apresentados, com base na técnica de Análise de Conteúdo de Lawrence Bardin, que compreende as seguintes etapas: pré-análise, exploração do material e tratamento dos resultados, inferência e a interpretação (BARDIN, 2011).

O estudo respeitou os preceitos éticos e foi aprovado pelo Comitê de Ética em Pesquisa com Seres Humanos da Universidade Federal de Viçosa, CAAE: 48394515.8.0000.5153.

\section{Resultados e discussão}

De forma geral, percebeu-se um grande interesse dos participantes pela dinâmica, colaborando para um maior envolvimento dos mesmos com o tema. Vale ressaltar que, para alguns, a temática era extremamente nova ou pouco conhecida, sendo essa informação consoante aos ditos de Gomes e Othero (2016), que afirmam que raramente a formação em cuidados paliativos é parte das matrizes curriculares dos cursos de graduação em saúde. Este fato evidencia a necessidade de investimentos em capacitações concernentes à temática junto a profissionais de saúde, o que se justifica ainda pelos dados da Worldwide Palliative Care Alliance (2014), que revelam que existe uma enorme demanda de pacientes necessitados desses cuidados, uma vez que menos de $8 \%$ destes têm de fato a assistência em cuidados paliativos garantida.

Os conceitos de cuidados paliativos trazidos pelos participantes foram organizados em três categorias, que podem ser visualizados na Figura 1.

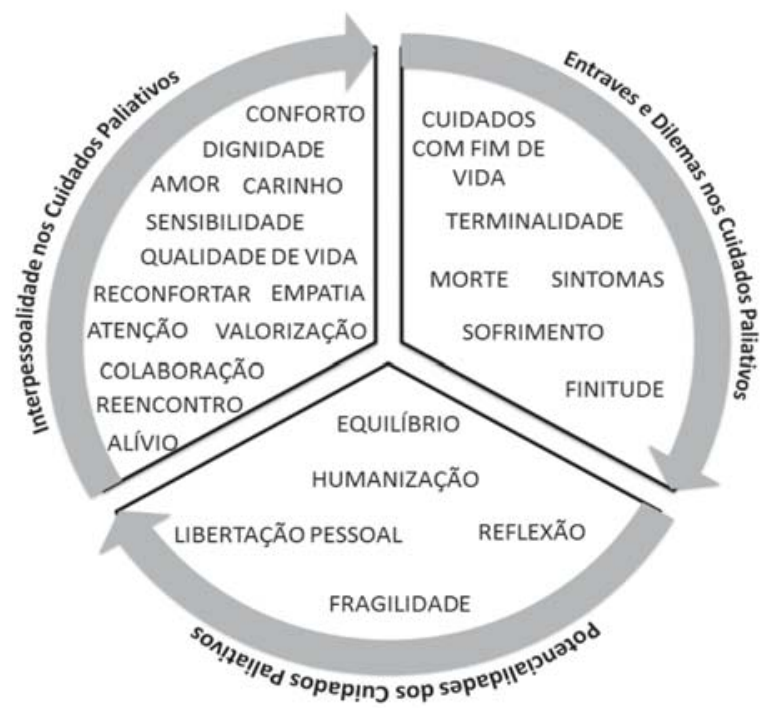

Figura 1 - Representação digital dos conceitos de cuidados paliativos, após Análise de Conteúdo. Fonte: Dados do estudo.

A primeira categoria, que foi intitulada "Interpessoalidade nos cuidados paliativos", revelou palavras que se relacionam com a interpessoalidade, estando, portanto, intimamente ligadas à relação com o outro. Ante os conceitos descritos pelos participantes do minicurso, foi possível perceber que os cuidados paliativos podem ser definidos por palavras que caracterizam as tecnologias leves, sendo elas: conforto, dignidade, alívio, carinho, sensibilidade, qualidade de vida, atenção, empatia, colaboração, reencontro, reconforto, valorização e amor. Merhy (1997) afirma que sendo o cuidar em saúde um processo vivo em ato, não é possível a exclusão dos sentimentos e emoções dos envolvidos, 
o que o autor denomina de tecnologia leve, pois requer baixa densidade tecnológica e se manifesta principalmente no universo das interações.

Nesse sentido, os cuidados paliativos têm por função fornecer qualidade de vida e conforto tanto à pessoa com câncer, como também ao seu núcleo familiar. E, para tanto, é necessária uma equipe multidisciplinar, preparada para atuar visando amenizar o sofrimento, dor e angústias por meio de estratégias de cuidado que abarquem também questões psicológicas e espirituais, auxiliando o núcleo familiar adoecido no enfrentamento da doença (HERMES e LAMARCA, 2013).

Por outro lado, na segunda categoria "Entraves e dilemas dos cuidados paliativos", as palavras e frases expressadas pelos participantes ao conceituarem os cuidados paliativos fizeram referência a aspectos negativos e a obstáculos enfrentados pelos indivíduos com câncer e suas famílias durante o tratamento, sendo elas: morte, sofrimento, finitude, cuidados com o fim da vida, terminalidade, sintomas.

Esses fatores podem ser associados ao estigma social que o câncer possui, sendo seu diagnóstico comumente associado à morte. Sendo assim, é comum que pessoas com câncer apresentem sentimentos como medo, ansiedade e angústia, e o tratamento em si é visto como uma batalha e sentença de final de vida (GARCIA, 2010; SALCI e MARCON, 2010).

É importante ressaltar, nesse interim, que um dos princípios dos cuidados paliativos é afirmar a importância da vida com dignidade, porém, sem desconsiderar a morte, que é um processo inerente a todos seres humanos, devendo ser vista como um processo natural (HERMES e LAMARCA, 2013).

Nessa perspectiva, a fundadora dos cuidados paliativos modernos, Cicely Saunders, argumenta que o profissional do cuidado deve se importar com a singularidade do paciente, até o último momento de vida deste, fazendo tudo o que estiver ao seu alcance, não somente para propiciar uma morte digna e em paz, mas também para oportunizar vida aos dias que lhe restam (SAUNDERS, 2013). Sendo assim, é de suma importância que os profissionais estejam capacitados para lidarem com o processo de morte e morrer, auxiliando não somente a pessoa com câncer, como também seus familiares no processo do luto.

Nesse contexto, a equipe multidisciplinar busca o bem-estar do ser cuidado e dos cuidadores, atuando no âmbito biopsicossocial do indivíduo, com vistas a amenizar a dor e proporcionando conforto. Desta forma, é possível que pessoas em processo de finitude passem por esse momento com dignidade e de forma natural (HERMES e LAMARCA, 2013). Assim, pensando na amplitude do conceito dos cuidados paliativos, que visam promover conforto e qualidade de vida em todas as fases do tratamento oncológico, há que se ressaltar também os aspectos positivos desta prática, visando destituir seu conceito como somente cuidados ao fim da vida ou no processo de morrer. É o que pode ser verificado na próxima categoria.

Nesta, denominada "Potencialidades dos cuidados paliativos", foi possível perceber que alguns participantes do minicurso o referem como uma prática positiva, que traz benefícios para os cuidadores. Esse fato pode ser evidenciado pela utilização de palavras como: humanização, equilíbrio, libertação pessoal, reflexão e fragilidade.

Tratando-se de cuidados paliativos, a Sociedade Brasileira de Geriatria e Gerontologia discorre que todos os envolvidos no cuidado requerem atenção, uma vez que lidam cotidianamente com múltiplas questões estressantes, tais como: sofrimento alheio, conflitos familiares, comunicação de más notícias, dentre outros. Assim, é fundamental um olhar atento para esses cuidadores, a fim de identificar evidências de sobrecarga. Contudo, vale ressaltar que ofertar cuidados paliativos também propicia ao cuidador o desenvolvimento de muitas competências (SBGG, 2014).

Kubler-Ross (2017) afirma que o processo de cuidar de pessoas amplamente debilitadas se equivale aos muitos anos de meditação de um monge, sendo possível, por meio desse processo, experimentar a compaixão e a empatia interconectadas, e com isso, ser levado ao equilíbrio, à libertação pessoal, à reflexão e à compreensão de sua própria fragilidade enquanto humano. Logo, a presente categoria se apresenta como uma potencialidade dos cuidados paliativos, visto que estes possibilitam o "estarcom". Santos, Pagliuca e Fernandes (2007) destacam que esse momento se apresenta como uma situação compartilhada, configurando-se como um compromisso existencial, visto que o cuidado se instituiu junto ao surgimento do homem.

Assim, essa experiência conduz ao acréscimo e ao desenvolvimento do potencial humano. Ante a isso, ressalta-se a frase do filosofo libanês Khalil Gibran, que afirma: "Do sofrimento emergiram os espíritos mais fortes, as personalidades mais sólidas, são marcadas com cicatrizes" (GIBRAN, 2019, p. 26). Logo, a 
presente categoria revela a via de mão dupla oportunizada pelos cuidados paliativos, uma vez que tanto o ser cuidado apresenta ganhos em seu processo de adoecimento, como também o cuidador tem a possibilidade de refletir e repensar sua própria condição humana.

Por fim, destaca-se a relevância de colocar em pauta e discussão temáticas como a de cuidados paliativos, visto que oportuniza aos estudantes e profissionais de saúde a troca de conhecimentos sobre o tema, além da (re)construção de conceitos e visões de mundo.

Sob essa perspectiva, a realização de oficinas, minicursos, dentre outras modalidades de práticas educativas sobre cuidados paliativos mostram-se relevantes no âmbito da saúde, e ferramentas estratégicas para o alcance de um cuidado humanizado, tendo em vista que existe uma carência mundial de profissionais qualificados nesta área.

\section{Conclusões}

Ainda existem muitos desafios na compreensão do que são os cuidados paliativos, e quando se trata da finitude da vida, muitos não estão preparados para discutir e/ou lidar com o processo de adoecimento e morte, sentindo-se incapazes diante de doenças que não possuem tratamento curativo.

Logo, é de suma importância a implementação de disciplinas e/ou estratégias de educação permanente que abordem os cuidados paliativos nas matrizes curriculares dos cursos da área da saúde, viabilizando espaços para discussão e disseminação dessa prática essencial na assistência à saúde. Há que se ressaltar ainda a importância de preparar profissionais que saibam lidar com o processo de morte e morrer, entendendo a morte como processo natural, e por fim, prestando uma assistência integral, pautada na humanização e fornecendo conforto e alívio da dor a pessoas com câncer, bem como aos seus familiares.

Ademais, é importante salientar a atuação estratégica da KARKINOS em promover espaços de discussão a respeito da temática abordada, tornando possível o contato de estudantes e profissionais da saúde e assegurando a capacitação de recursos humanos para atuação na área oncológica.

\section{Referências}

BARDIN, L. Análise de conteúdo. Lisboa: Edição 70, 2011.

DELALIBERA, M. et al. Sobrecarga no cuidar e suas repercussões nos cuidadores de pacientes em fim de vida: revisão sistemática da literatura. Ciência E Saúde Coletiva, Rio de janeiro, v. 20, n. 9, p. 2731-2747, set. 2016.

GARCIA, C. C. O câncer na FSP: mitos, divulgação científica e construção social da notícia. Revista Científica Integrada, Ribeirão Preto, v. 4, n. 7, p. 20-34, jul. 2010.

GIBRAN, K. The Prophet. São José: Independently Published, 2019.

GOMES, A. L. Z.; OTHERO, M. B. Cuidados paliativos. Estudos Avançados. São Paulo, v. 30, n. 88, p. 155-166, dez. 2016.

HERMES, H. R.; LAMARCA, I. C. A. Cuidados paliativos: uma abordagem a partir das categorias profissionais de saúde. Ciência \& Saúde Coletiva, Rio de Janeiro, v. 18, n. 9, p. 2577-2588, set. 2013.

IAHPC - International Association for Hospice e Palliative Care - Palliative Care Definition. Disponível em: https://hospicecare.com/what-we-do/projects/consensus-based-definition-ofpalliative-care/definition/. Acesso em 20 de mar. 2019.

INCA - Instituto Nacional de Câncer. Brasil. Estimativa 2018/2019: incidência de câncer no Brasil. Rio de Janeiro: INCA, 2018.

KUBLER-ROSS, E. A roda da vida. Rio de Janeiro: Sextante, 2017.

MERHY, E. E. Em busca do tempo perdido: a micropolítica do trabalho vivo em saúde. Em:

MERHY, E. E.; ONOCKO, R. Agir em saúde. Um desafio para o público. São Paulo: Editora Hucitec / Buenos Aires: Lugar Editorial, 1997. 
OMS. Organização Mundial da Saúde. Guide to cancer early diagnosis. Geneva: World Health Organization; 2017. Disponível em: http://apps.who.int/iris/bitstream/handle/10665/254500/ 9789241511940-eng.pdf;jsessionid=88544CA533902AAB691C5AFCE32EC10C? sequence=1. Acesso em 20 de mar. 2019.

SALCI, M. A.; MARCON, S. S. A convivência com o fantasma do câncer. Revista Gaúcha de Enfermagem, v. 31, n. 1, p. 18-25, 2012.

SANTOS, M. C. L.; PAGLIUCA, L. M. F.; FERNANDES, A. F. C. Cuidados paliativos ao portador de câncer: reflexões sob o olhar de Paterson e Zderad. Revista Latino-Americana de Enfermagem, Ribeirão Preto, v. 15, n. 2, p. 350-354, mar./abr, 2007.

SAUNDERS, C. Velai comigo - inspiração para uma vida em Cuidados Paliativos. Lisboa: Universidade Católica Editora, 2013.

SBGG - Sociedade Brasileira de Geriatria e Gerontologia. Vamos falar de cuidados paliativos. Rio de Janeiro: SBGG, 2014.

WORLDWIDE PALLIATIVE CARE ALLIANCE. Global Atlas of Palliative Care at the End of Life. WHO. England. 2014. Disponível em: http://www.who.int/nmh/

Global_Atlas_of_Palliative_Care.pdf. Acesso em 20 de mar. 2019.

Data de submissão: 30/3/2019. Data de aceite: 29/4/2019. 